\title{
Tumor after tumor in two years: A rare presentation of brain glioma, meningioma and supraclavicular meningiosarcoma
}

\author{
Riana Kipiani Abdul Halim, Mohd. Solahuddin Mohd Kenali, \\ Primuharsa Putra Sabir Husin Athar, BS Gendeh, \\ Wan Muhaizan Wan Mustaffa, Halili Rahmat
}

\begin{abstract}
Introduction: Low-grade gliomas (LGG) and meningioma are common brain tumors but it is rare for both tumors to be diagnosed in a same patient. Furthermore, it is rare for the same patient to develop an extracranial primary or metastasis meningioma. Probably this is the first case report of a second primary with extracranial meningiosarcoma in literature. Case Report: We present a rare case of brain atypical meningioma which occurred shortly following craniotomy
\end{abstract}

Riana Kipiani Abdul Halim¹, Mohd. Solahuddin Mohd Kenali $^{2}$, Primuharsa Putra Sabir Husin Athar ${ }^{3}$, BS Gendeh ${ }^{4}$, Wan Muhaizan Wan Mustaffa ${ }^{5}$, Halili Rahmat ${ }^{6}$

Affiliations: ${ }^{1} \mathrm{MD}$, Resident, Otorhinolaryngology- Head and Neck Surgery department, KPJ Healthcare University College, Negeri Sembilan, Malaysia; ${ }^{2} \mathrm{MD}$, B.MED.SC, MS (ORL-HNS), Fellowship Head and Neck, Consultant Ear, Nose, Throat/ Head \& Neck Surgeon, KPJ Tawakkal Specialist Hospital, Kuala Lumpur, Malaysia and KPJ Healthcare University College, Negeri Sembilan, Malaysia; ${ }^{3} \mathrm{MD}$, MS (ORL-HNS), Ear, Nose, Throat/ Head \& Neck Consultant Clinic, KPJ Seremban Specialist Hospital, Negeri Sembilan, Malaysia and KPJ Healthcare University College, Negeri Sembilan, Malaysia; ${ }^{4}$ MBBS, MS (ORL-HNS), Consultant Surgeon and Lecturer Department of Otorhinolaryngology, Head and Neck Surgery, Universiti Kebangsaan Malaysia Medical Centre, Kuala Lumpur, Wilayah Persekutuan K.L, Malaysia; ${ }^{5} \mathrm{MD}$, MPath, ISN Fellow, Consultant Histopathologist, Lablink Medical Laboratory, Kuala Lumpur, Malaysia; ${ }^{6}$ MBBS, FRACS, Neurosurgery Clinic, KPJ Tawakkal Specialist Hospital, Kuala Lumpur, Malaysia.

Corresponding Author: Primuharsa Putra Sabir Husin Athar KPJ Seremban Specialist Hospital, Jalan Toman 1, Kemayan Square, Seremban, Negeri Sembilan, Malaysia, 70200; Email: putrani@yahoo.co.uk

Received: 25 March 2016

Accepted: 18 May 2016

Published: 08 June 2016 and radiotherapy after being diagnosed as brain glioma (diffused astrocytoma) in a 22-year-old Malay female. Tumors occurred at different sites of the brain namely the atypical meningioma at the temporal and the initial glioma at the frontal region. Subsequently, patient developed a right supraclavicular meningiosarcoma. We believe that the patient could have developed radiationinduced meningioma but the transformation period is very short. The patient's mother had history of brain tumor that added into the risk factors in this patient. However, we are unable to confirm whether the supraclavicular mass was a primary supraclavicular meningiosarcoma or metastatic meningiosarcoma progression from atypical meningioma. Conclusion: There are multiple variant revealed associations of glioma and meningioma. However, these variant could be coincidental. There is a possibility that one tumor acts locally as an irritating factor that induced another unrelated neoplasm. Relation of two or more types of cancers might lead to new therapies, provided target surveillance for cancer patients and risk factors or causal agent are identified.

Keywords: Astrocytoma, Meningioma, Meningiosarcoma, Second primary, Seizure

\section{How to cite this article}

Halim RKA, Mohd Kenali MS, Athar PPSH, Gendeh BS, Wan Mustaffa WM, Rahmat H. Tumor after tumor in two years: A rare presentation of brain glioma, meningioma and supraclavicular meningiosarcoma. Case Rep Int 2016;5:22-26.

\section{Article ID: 100025CRINTRH2016}


doi:10.5348/crint-2016-25-CR-6

\section{INTRODUCTION}

Gliomas are primary brain tumors of the central nervous system. They account approximately $30 \%$ of all brain and central nervous system tumors and $80 \%$ of all malignant brain tumors [1].

Meningioma is a tumor that arises from the arachnoidal cap cells of the brain and spinal cord meninges. There are three grades for meningioma and has multiple variants for each type by World Health Organization (WHO). Meningothelial, fibrous (fibroblastic), transitional (mixed), psammomatous, angiomatous, microcytic, secretory, lymphoplasmocyterich and metaplastic are examples from grade I. Grade II examples are clear cell, chordoid and atypical rhabdoid, papillary and anaplastic (malignant), can be in a form of sarcoma or carcinoma and are grade III [2].

Extracranial meningioma is very rare. Primary extracranial meningioma account less than $2 \%$ from all types of meningioma in a large series in the United States [3]. Secondary meningioma or distant metastasis are very rare, only $0.1 \%$ of incidence [4].

There have been reported of 111 cases of coincidental intracranial meningioma and glioma in a literature. The condition is very rare and there will be a diagnostic dilemma as well as difficulty in management [5]. We reported a rare occurrence of a meningioma following total excision and radiotherapy of glioma in a short period. Herein, an atypical meningioma is transformed into meningiosarcoma and metastasized extracranially in a month. There is also a possibility but very rare of this patient to develop primary extracranial meningiosarcoma.

Two or more primary cancer can coexist at the time of diagnosis (synchronous), or develop consequently (metachronous), sometimes years after resection of the first primary. There are multiple reasons reported for the occurrence of two primary cancers in short duration period. Both synchronous or metachronous cancer may be related and share the same factors. If the cells were the same type, it can indicate metastasis, but, however, both cancers might be independent. The time of diagnoses of both cancers are also important to determine whether the second cancer developed from the first cancer treatment or the natural age presentation of the second cancer. Relation of two or more types of cancers might lead to new therapies, provided target surveillance for cancer patients risk factors or causal agent are identified [6].

\section{CASE REPORT}

A 20-year-old Malay female was referred to our centre in May 2013. She presented with fever for 2 days, vomiting and right diplopia. Patient's mother had history of brain tumor, with no further details available. Physical findings were right ptosis with sluggish pupillary reaction. Other findings were unremarkable. The CT scan and the MRI scan of brain were reported as right frontal lobe tumor

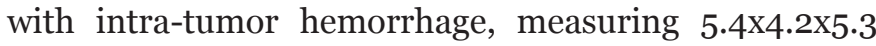
$\mathrm{cm}$ (Figure 1A). She underwent craniotomy and tumor resection followed by radiotherapy. The histopathology revealed diffused astrocytoma grade 2 (Figure 1B).

She presented with recurrent headaches in December 2014. Patient had multiple follow-ups from first craniotomy to this presentation in 1 year 7 months. During follow-up patient was well. CT brain showed right temporal brain tumor (Figure 2A). Patient underwent craniotomy followed by radiotherapy. Histopathology was reported as atypical meningioma (Figure 2B).

One month after second craniotomy, she presented with right supraclavicular swelling which was gradually increasing in size. and associated with right shoulder and arm pain, weakness and numbness. There was no skin changes, it was fixed, firm and measured around 6 $\mathrm{cm}$ in diameter. No other swellings were noted. Her right power grip was $4 / 5$ and other neurological assessments were unremarkable. Radial pulse was normal. Other examinations were unremarkable.

A CT scan brain revealed multiple intracranial enhancing mass $(3.1 \times 2.2 \mathrm{~cm})$ at the craniotomy site at frontal and parietal region with minimal midline shift to the left and dilated lateral ventricles. The CT scan of supraclavicular mass showed irregular wall enhancement $(5.0 \times 3.6 \mathrm{~cm} \times 5.6 \mathrm{~cm})$ with a hypodense centre suggesting necrosis. There was no evidence of lung masses (Figure 3A).
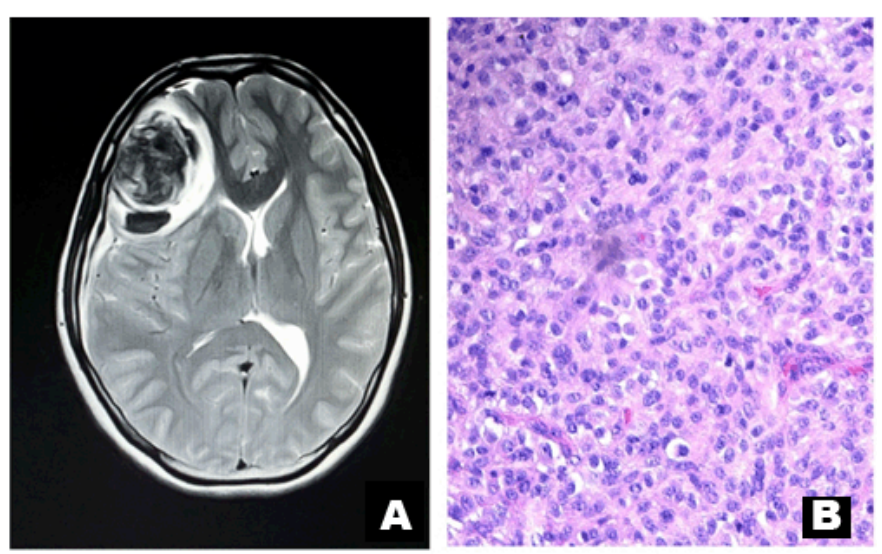

Figure 1: Diffused astrocytoma grade 2. (A) An axial cut of MRI brain with gadolinium contrast, revealed a large cortical based tumor $5.0 \times 4.1 \times 3.7 \mathrm{~cm}$ demonstrated in the right frontal lobe with a large area of possibility of intratumoral hemorrhage/ calcification. There is perilesional edema with mass effect causing effacement of right lateral ventricle and midline shift. (B) Right frontal mass mildly enlarged, pleomorphic, hyperchromatic nuclei with moderate cytoplasm. Some cells reveal prominent nucleoli and have gemistocytic features with occasional mitosis seen (H\&E stain, x100). 
Fine needle aspiration of the supraclavicular mass was inconclusive. Exploration and debulking of the supraclavicular mass (April 2015) revealed it being adherent to the adjacent tissue. Superomedially to the scalene muscle and inferolaterally to the chest wall. Histopathological examination was reported as malignant tumor with sarcomatous appearance (Figure 3B). Immunochemistry tests revealed features that were compatible of meningiosarcoma or malignant meningioma. It was positive to epithelial membrane antigen (EMA), vimentin, S1Oo and focal positive with glial fibrillary acidic protein (GFAP). No brain biopsy was performed.

Patient has multiple risk factors of brain tumor namely being a female in the reproductive age with a family history of brain tumor (mother) and had history of radiation.

She was referred to the oncologist for further treatment but defaulted follow-up. She was then readmitted in July 2015 due to swelling on the scalp over the post craniotomy site (Figure 4). However, no further active intervention was done at this stage.

\section{DISCUSSION}

Gliomas are more common with increasing age, male, white race and non-Hispanic ethnicity. Gliomas causes are unknown. However, families with neurofibromatosis, a genetic inherited disorder, have increased risk of developing gliomas. WHO grade I and II are considered low-grade. Low-grade astrocytomas are uncommon tumors compared to higher-grade III and IV. LGGs especially diffuse astrocytoma typically arises in young adults as reported in this case [1].

Diffuse astrocytoma should not be considered benign even though it is a slow growing tumour. "Watchful waiting" with MRI scanning is recommended for lesions
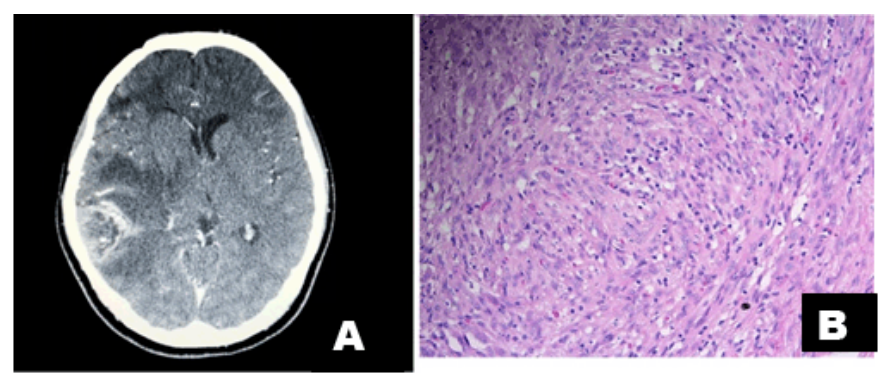

Figure 2: Atypical meningioma (A) image is an axial cut of patient CT scan brain post contrast. Report revealed right temporoparietal mass $2.8 \times 3.1 \times 2.9 \mathrm{~cm}$. Irregular mass with areas of necrosis. Midline shift to the left. No more right frontal mass. (B) Right temporal tissue revealed the tumor cells arranged in cohesive sheets arrangement. The tumor cells are spindle in shape with oval to slender nuclei, moderate cytoplasm, have indistinct border and abundant fibrocollagenous bundles. The mitotic count is 8 in 10 (hpf) (H\&E stain, x200).
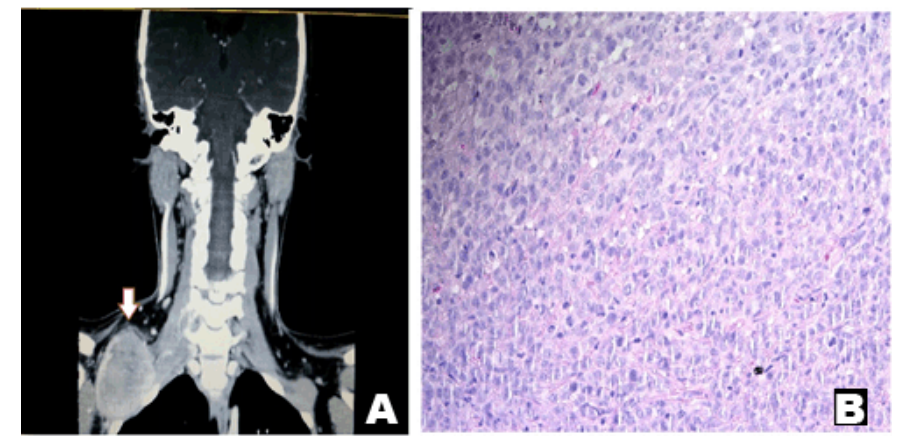

Figure 3: Right supraclavicular mass meningiosarcoma. (A) A coronal view of CT scan head and neck post contrast. There is a large supraclavicular mass lies in the intramuscular fat measuring $5.0 \times 3.6 \times 5.6 \mathrm{~cm}$. Irregular mass with hypodense center suggesting as necrosis area. (B) Right supraclavicular mass tissue composed of cellular compact short spindle cells with areas of necrosis. The malignant cells showed enlarge pleomorphic round to oval nuclei and some with inclusion. There is also increase mitosis $24 / \mathrm{hpf}$ in bizarre form (H\&E stain, x200).

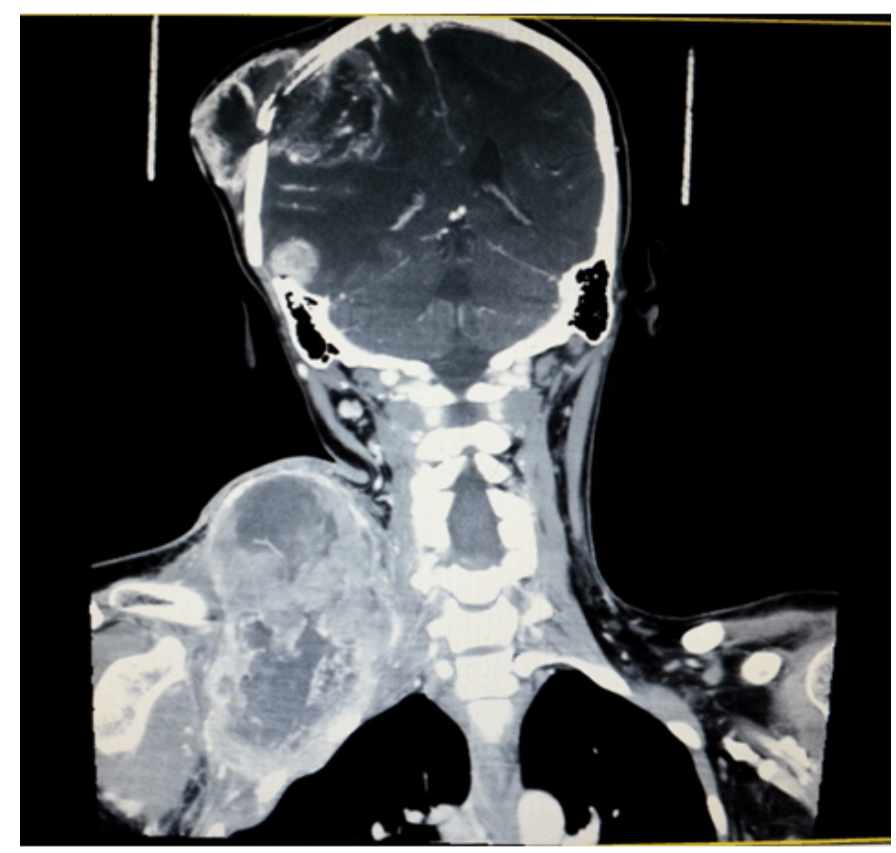

Figure 4: A coronal view of computed tomography scan of head and neck post-contrast. The scan reported as multiple tumor recurrences in the cerebral hemisphere with significant white matter edema, mass effect and midline shift. Large soft tissue mass within the right supraclavicular fossa.

with surgical inaccessibility, well-controlled symptoms of seizure with anticonvulsants and patients not keen for surgery. Conventional strategies may include surgery, radiotherapy, chemotherapy or combinations. Overall survival has significant improvement in more aggressive resection compared to debulking [1].

This patient was re-admitted with another brain tumor which was revealed as atypical meningioma, a grade II meningioma. There have been similar reports in an intracranial collision tumor which have histologically distinct primary tumors such as meningioma and glioma 
in the same brain region. However in this case, the tumors occurred in different sites of the brain at different times.

In general, meningioma is more common in women. Endogenous and exogenous hormones could be considered as one of the factor predisposing to meningioma. Meningioma tends to grow larger in pregnancy and during the luteal phase of menstruation period [2].

Radiation also is one of the risk factors pre-disposing to radiation-induced meningioma [2]. Reported risks of developing meningioma, 6-10 times higher in exposure to ionizing radiation than no exposure [7]. Radiationinduced meningioma is a known long-term complication of radiation exposure. A retrospective review of 30 patients with radiation-induced meningioma within 1960 to 2010 had mean radiation dose was 34 Gy (range 16-6o Gy). The review also showed that the latency time to meningioma was 26 years with the range of 8 to 51 years [8-10]. However, in our case the duration between the first radiation and development of meningioma was 1 year and 7 months. There is also increased risk of meningioma in certain genetic diseases such as in neurofibromatosis (NF type I and II) and familial meningioma [2].

Management of meningioma according to National Comprehensive Cancer Network guidelines 2011, are based on patients symptoms and size of tumor [2]. In asymptomatic patients with small tumor, active surveillance must be done to observe patients signs and symptoms and the tumor progression. In an accessible tumor, surgery is indicated for adequate removal of the tumor and surrounding tissue. For tumor that cannot be fully removed during surgery or recurrence, the treatment plan often includes radiation therapy and/or chemotherapy post-surgery $[2,5]$.

Atypical meningioma has aggressive behavior, increased risks of recurrence and a small risk of distant metastasis. Studies have shown that the recurrence rate were dependent on the extent of surgical resection, tumor location and accessibility of the tumor and the involvement of dura mater and bone [4].

Histopathological and immunochemistry test of the supraclavicular mass was reported as meningiosarcoma or malignant meningioma. It could have metastasized from a hematogenous spread of blood-brain barrier breached though previous craniotomy or by extra neural invasion of possible cervical plexus. In intracranial meningioma, 20\% were reported to have extended to extradural space, whether via direct extension or metastasis [2]. Incidence of metastasis in meningioma has been reported to be less than 1 per $1,000[2,8]$. In all types of meningiomas, the incidence metastasis is $0.76 \%$, unlike malignant meningioma which has $43 \%$ incidence [8]. Local recurrence ranges from 9 to $32 \%$ following a complete tumour resection. The incidence of metastatic meningioma of atypical type and malignant meningioma are $5 \%$ and $30 \%$ respectively [4].
However, primary extradural meningiomas are more rare. It has been reported that extradural meningiomas occurs in a variety locations, more common in head and neck. There are association of extracranial meningioma with other neoplasm such as von Recklinghausen's neurofibromatosis, tuberous sclerosis, osteoma, and ovarian fibromas. There are no definitive management of extracranial meningioma reported in literature [2].

Literature reviews has confirmed the association of intracranial meningiomas and gliomas. However, it is well recognized that the association of meningioma and glioma in the same patient represent a rare event. In young females endogenous and exogenous hormone could be a contributing factor. However, several authors reported that these associations are considered purely coincidental [5].

Prognosis of metastasis meningioma at the median follow up time of 24 months was that $92 \%$ were alive. This comprises of $95 \%$ benign and $78 \%$ malignant. Twenty four per cent had tumor recurrences which were $22 \%$ low grade and $33 \%$ high grade [2].

\section{CONCLUSION}

Following resection and radiotherapy of initial brain tumor, it is possible that this patient developed radiation-induced meningioma or maybe recurrence of the meningioma. It due time it may have progressed to malignant meningioma or meningiosarcoma which eventually metastasized to the supraclavicular region. There is also possibility that one tumor acts locally as an irritating factor that induced another unrelated neoplasm. Relation of two or more types of cancers might lead to new therapies, provided target surveillance for cancer patients and risk factors or causal agent are identified.

$$
* * * * * * * * *
$$

\section{Acknowledgements}

We acknowledge all the Radiologists in KPJ Tawakkal Specialist Hospital, Kuala Lumpur, Wilayah Persekutuan K.L, Malaysia that involved in the reporting of all the images.

\section{Author Contributions}

Riana Kipiani Abdul Halim - Substantial contributions to conception and design, Acquisition of data, Analysis and interpretation of data, Drafting the article, Revising it critically for important intellectual content, Final approval of the version to be published

Mohd. Solahuddin Mohd Kenali - Analysis and interpretation of data, Revising it critically for important intellectual content, Final approval of the version to be published

Primuharsa Putra Sabir Husin Athar - Analysis and interpretation of data, Revising it critically for important 
intellectual content, Final approval of the version to be published

BS Gendeh - Analysis and interpretation of data, Revising it critically for important intellectual content, Final approval of the version to be published

Wan Muhaizan Wan Mustaffa - Analysis and interpretation of data, Revising it critically for important intellectual content, Final approval of the version to be published

Halili Rahmat - Analysis and interpretation of data, Revising it critically for important intellectual content, Final approval of the version to be published

\section{Guarantor}

The corresponding author is the guarantor of submission.

\section{Conflict of Interest}

Authors declare no conflict of interest.

\section{Copyright}

(C) 2016 Riana Kipiani Abdul Halim et al. This article is distributed under the terms of Creative Commons Attribution License which permits unrestricted use, distribution and reproduction in any medium provided the original author(s) and original publisher are properly credited. Please see the copyright policy on the journal website for more information.

\section{REFERENCES}

1. Raizer J, A Parsa A. Current Understanding and Treatment of Gliomas. Cancer Treatment and Research 2015;163-201.
2. Joung HL. Meningiomas: Diagnosis, Treatment, and Outcome. London, United Kingdom: Springer-Verlag London Ltd; 2008.

3. Rushing EJ, Bouffard JP, McCall S, et al. Primary extracranial meningiomas: an analysis of 146 cases. Head Neck Pathol 2009 Jun;3(2):116-30.

4. Lee GC, Choi SW, Kim SH, Kwon HJ. Multiple extracranial metastases of atypical meningiomas. J Korean Neurosurg Soc 2009 Feb;45(2):107-11.

5. Spallone A, Santoro A, Palatinsky E, Giunta F. Intracranial meningiomas associated with glial tumours: a review based on 54 selected literature cases from the literature and 3 additional personal cases. Acta Neurochir (Wien) 1991;110(3-4):133-9.

6. Bajdik CD, Abanto ZU, Spinelli JJ, Brooks-Wilson A, Gallagher RP. Identifying related cancer types based on their incidence among people with multiple cancers. Emerg Themes Epidemiol 2006 Nov 8;3:17.

7. Claus EB, Calvocoressi L, Bondy ML, Schildkraut JM, Wiemels JL, Wrensch M. Family and personal medical history and risk of meningioma. J Neurosurg 2011 Dec;115(6):1072-7.

8. Estanislau ES, Carvalho GT, Reis BL, et al. Malignant meningioma with extracranial metastases. Arq Neuropsiquiatr 2009 Sep;67(3A):730-2.

9. Zhang D, Yu J, Guo Y, Zhao S, Shao G, Huang H. An intraventricular meningioma and recurrent astrocytoma collision tumor: a case report and literature review. World J Surg Oncol 2015 Feb 12;13:37.

10. Morgenstern PF, Shah K, Dunkel IJ, et al. Meningioma after radiotherapy for malignancy. $\mathrm{J}$ Clin Neurosci 2016 Apr 8. pii: So967-5868(16)00079-5.
Access full text article on other devices

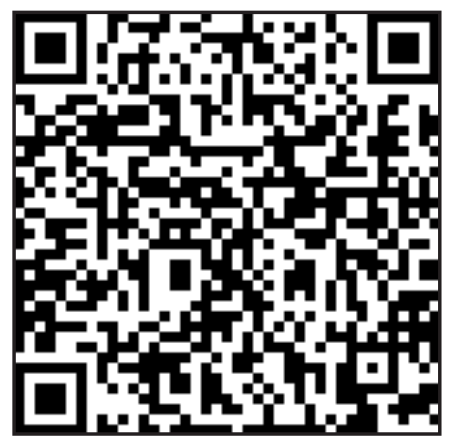

Access PDF of article on other devices

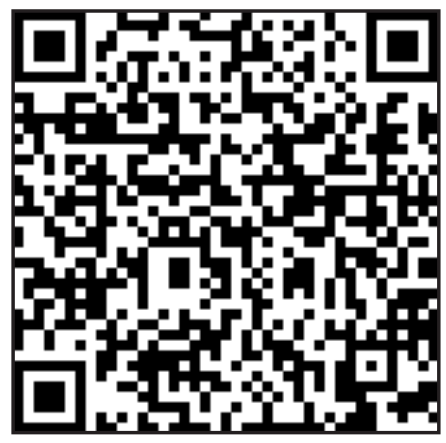

